\title{
Spontaneous Resolution of Delayed Onset Large Subclavian Artery Pseudoaneurysm -Case Report-
}

\author{
Tsuyoshi OHTA, Hidetsugu MAEKAWA, Kenji KASUNO*, \\ Tatsuo TSUKAMOTO*, Eri MUSO*, and Masatsune ISHIKAWA \\ Departments of Neurosurgery and ${ }^{*}$ Nephrology \& Dialysis, Kitano Hospital, Osaka
}

\begin{abstract}
A 70-year-old woman with a history of end-stage renal disease secondary to anti-neutrophil cytoplasmic autoantibody-associated vasculitis had been receiving hemodialysis for 5 years. The patient underwent attempted right internal jugular vein cannulation for temporary hemodialysis catheter placement. Pulsating mass developed in the neck and angiography revealed a subclavian artery pseudoaneurysm 4 days later. The pseudoaneurysm disappeared spontaneously during the interval between the diagnosis and the planned surgical procedure. Such delayed onset and spontaneous resolution of subclavian artery pseudoaneurysm is uncommon. Close observation may be optimal if delayed onset of pseudoaneurysm occurs after small needle puncture with cessation of antiplatelet/anticoagulant administration.
\end{abstract}

Key words: subclavian artery pseudoaneurysm, spontaneous resolution, antiplatelet, angiography, renal failure

\section{Introduction}

Subclavian artery pseudoaneurysm is a rare but severe

Received February 13, 2009;

Accepted April 13, 2009 complication of internal jugular puncture, usually occurring with sudden onset, progressive nature, and high risk of death. ${ }^{2,8,14)}$ Here, we describe a case of subclavian artery pseudoaneurysm with delayed onset and spontaneous resolution. 


\section{Case Report}

A 70-year-old woman with a history of end-stage renal disease secondary to anti-neutrophil cytoplasmic autoantibody-associated vasculitis had been receiving hemodialysis for 5 years. The arteriovenous (AV) shunt in her left forearm had become stenotic twice, and was treated both times with percutaneous transluminal angioplasty (PTA). Aspirin $100 \mathrm{mg}$ was administered for the prevention of restenosis. She was admitted to our hospital for third revision of the AV shunt.

Catheterization of the right internal jugular vein for temporary hemodialysis catheter placement was attempted several times but was unsuccessful (Day 0). Manual compression of the right side of her neck was continued for 40 minutes because of arterial puncture with a 19 gauge needle. No apparent active bleeding persisted. Another catheter was placed in the femoral vein.

Four days later, she noticed a pulsating mass developing in the right side of her neck. Color Doppler ultrasonography showed a pulsating mass of $25.6 \times 33.3$ $\times 29.6 \mathrm{~mm}$, and turbulent flow lateral to the right common carotid artery and just above the subclavian artery. Angiography using nonionic iodinated contrast medium (iomeprol) on Day 5 revealed a pseudoaneurysm around the right subclavian artery with poor clearance of iomeprol. Based on the delayed expansion, pseudoaneurysm of some branch of the subclavian artery was suspected. The patient and her family first requested conservative treatment. Hemodialysis was performed with nafamostat mesilate from Day 1. Aspirin administration was stopped on Day 4. She complained of gradual exacerbating pain over her neck and dysesthesia in her right shoulder, although the mass remained the same size.

Coil embolization of the feeding artery was attempted on Day 11. Superselective angiography from the thyrocervical trunk detected no connection with the pseudoaneurysm. Three-dimensional rotational angiography using an Allura Xper FD20/10 and processed by Integris 3DRA (Philips Medical Systems Nederland B.V., Best, The Netherlands) revealed a small opening on the dorsosuperior side of the subclavian artery (Fig. 1). The mass shape had changed from spherical to ellipsoid and the outflow had become slower. Coil embolization was abandoned because of the direct origin of the pseudoaneurysm from the subclavian artery. Cerebral angiography was performed to ascertain the feasibility of covered stenting over the opening, and revealed hypoplastic left vertebral artery and no cross flow from the left anterior communicating artery, even with compression of the right common carotid artery. Covered stenting might cause occlusion of the right vertebral artery, because of the short distance between the opening of the pseudoaneurysm and the origin of the right vertebral artery (less than $3 \mathrm{~mm}$ ), which would surely result in massive infarction in the posterior fossa. Therefore, open surgery was planned.

She reported alleviation of the pain on Day 12. The mass had apparently decreased in size and no pulsation was felt on Day 14. Ultrasonography did not show the pseudoaneurysm or mass. Her complaints had completely disap-

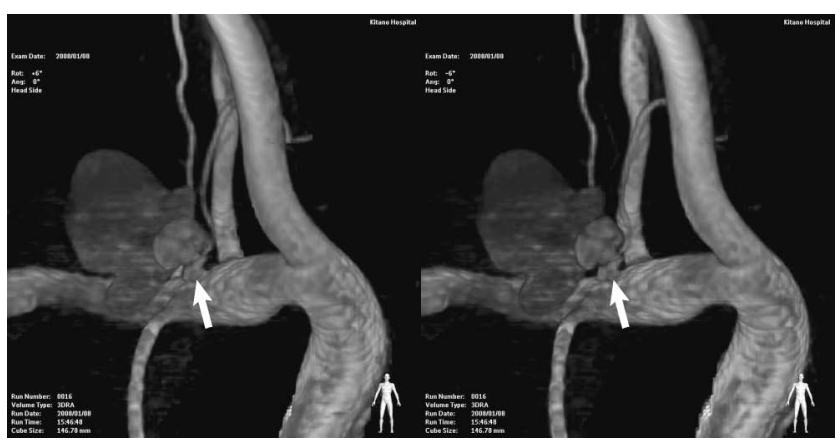

Fig. 1 Three-dimensional rotational angiograms seen from in front clearly depicting the orifice (arrows), configuration, and shape of the pseudoaneurysm and the adjacent branches. These stereographic images should be viewed by the cross fusion method.

peared. PTA for the existing AV shunt was performed and she was discharged without further intervention or sequelae.

\section{Discussion}

Serious complications associated with central venous access occur in $0.4-9.9 \%$ of patients undergoing attempted central venopuncture, ${ }^{11)}$ but inadvertent arterial puncture with a small needle is usually benign, and occurs in $0 \%$ to $11 \%$ of cases. ${ }^{6}$ Delayed development of pulsating mass in the supraclavicular region is often associated with pseudoaneurysm of the thyrocervical trunk ${ }^{16)}$ or transverse cervical artery. ${ }^{4}$

Arterial trauma caused by a large-bore dilator or catheter is usually associated with serious complications and requires prompt surgical or endovascular treatment. ${ }^{6}$ Coil placement into a pseudoaneurysm in the acute phase can be dangerous because of the absence of a true aneurysm wall. Therefore, endovascular occlusion of the parent vessel is often the most effective treatment if possible. ${ }^{7)}$ Endovascular treatment of a pseudoaneurysm of the subclavian artery with stented graft was reported, ${ }^{14)}$ but occlusion of the parent artery was as high as $16.6 \%$ with placement of covered stent-grafts in peripheral nonatherosclerotic arterial lesions. ${ }^{17)}$ In the present patient, occlusion or even stenosis of the right subclavian artery will exert an unfavorable influence on creation of the right forearm AV shunt in the future. The collateral flows through the circle of Willis and the contralateral vertebral artery must also be first estimated.

Spontaneous resolution of pseudoaneurysm sometimes occurs in the femoral artery, ${ }^{10)}$ pulmonary artery, ${ }^{19)}$ middle meningeal artery, ${ }^{18)}$ and other arteries. ${ }^{9}{ }^{9}$ Pseudoaneurysm often occurs in the arteries with a superficial course, with rather small calibers, and/or injury with a small needle. Stopping of aspirin administration, ${ }^{1,13,15)}$ change from unfractionated heparin to nafamostat mesilate, ${ }^{12)}$ and/or thrombogenicity of nonionic iodinated contrast medium ${ }^{3)}$ might promote aneurysmal thrombosis, but spontaneous resolution of unknown etiology is most likely. A period of 
observation is often recommended with the hope that thrombosis and resolution of the pseudoaneurysm might occur. ${ }^{10)}$

Only two-dimensional ultrasonography is necessary to confirm the diagnosis of pseudoaneurysm, but angiography is essential for evaluation of the aneurysm origin and the neighboring cerebral vasculature to plan the treatment. Selective injection is often necessary to determine the feeder branches. ${ }^{5}$ In the present case, conventional angiography was inadequate to define the true opening, and three-dimensional rotational angiography was superior for depicting the stereoscopic configuration of the pseudoaneurysm and the adjacent branches. Computed tomography angiography may be a valuable tool for follow-up examination. ${ }^{17)}$

Even delayed development of a pulsating mass after attempted internal jugular vein catheterization might be due to subclavian artery pseudoaneurysm. Aggressive treatment such as covered stent or surgical ligation is the treatment of choice, but spontaneous resolution might happen. Three-dimensional rotational angiography is useful for depicting the details of the pseudoaneurysm and the adjacent branches.

\section{References}

1) Ali M, McDonald JW, Thiessen JJ, Coates PE: Plasma acetylsalicylate and salicylate and platelet cyclooxygenase activity following plain and enteric-coated aspirin. Stroke 11: 9-13, 1980

2) Baldwin RT, Kieta DR, Gallagher MW: Complicated right subclavian artery pseudoaneurysm after central venipuncture. Ann Thorac Surg 62: 581-582, 1996

3) Chronos NA, Goodall AH, Wilson DJ, Sigwart U, Buller NP: Profound platelet degranulation is an important side effect of some types of contrast media used in interventional cardiology. Circulation 88: 2035-2044, 1993

4) Cuhaci B, Khoury P, Chvala R: Transverse cervical artery pseudoaneurysm: a rare complication of internal jugular vein cannulation. Am J Nephrol 20: 476-482, 2000

5) Dwivedi AJ, Cherukupalli C, Dayal R, Krishansastry KV: Endovascular treatment of false aneurysm of the thyrocervical trunk. Vasc Endovascular Surg 41: 77-79, 2007

6) Guilbert MC, Elkouri S, Bracco D, Corriveau MM, Beaudoin N, Dubois MJ, Bruneau L, Blair JF: Arterial trauma during central venous catheter insertion: Case series, review and proposed algorithm. J Vasc Surg 48: 918-925, 2008

7) Hopkins JK, Shaibani A, Ali S, Khawar S, Parkinson R, Futterer S, Russell EJ, Getch C: Coil embolization of posttraumatic pseudoaneurysm of the ophthalmic artery causing subarachnoid hemorrhage. Case report. J Neurosurg 107:
1043-1046, 2007

8) Huddy SP, McEwan A, Sabbat J, Parker DJ: Giant false aneurysm of the subclavian artery. An unusual complication of internal jugular venous cannulation. Anaesthesia 44: 588-589, 1989

9) Ibáñez Pradas V, García Vila JH, Redondo Ibáñez M, Díaz Ramón C: Spontaneous resolution of intrarenal pseudoaneurysm. Eur J Pediatr Surg 16: 362-364, 2006

10) Johns JP, Pupa LE Jr, Bailey SR: Spontaneous thrombosis of iatrogenic femoral artery pseudoaneurysms: documentation with color Doppler and two-dimensional ultrasonography. J Vasc Surg 14: 24-29, 1991

11) Koklu E, Poyrazoglu H, Yikilmaz A, Canpolat M, Konuskan B: Subclavian artery pseudoaneurysm: a rare and serious complication of central venous catheterization in an infant. Pediatr Radiol 38: 220-223, 2008

12) Matsuo $T$, Kario $K$, Nakao $K$, Yamada T, Matsuo M: Anticoagulation with nafamostat mesilate, a synthetic protease inhibitor, in hemodialysis patients with a bleeding risk. Haemostasis 23: 135-141, 1993

13) O'Brien JR: Effects of salicylates on human platelets. Lancet 1(7546): 779-783, 1968

14) Pastores SM, Marin ML, Veith FJ, Bakal CW, Kvetan V: Endovascular stented graft repair of a pseudoaneurysm of the subclavian artery caused by percutaneous internal jugular vein cannulation: case report. Am J Crit Care 4: 472-475, 1995

15) Patrono C, Coller B, FitzGerald GA, Hirsh J, Roth G: Plateletactive drugs: the relationships among dose, effectiveness, and side effects: the Seventh ACCP Conference on Antithrombotic and Thrombolytic Therapy. Chest 126(3 Suppl): 234S-264S, 2004

16) Peces R, Navascués RA, Baltar J, Laurés AS, Alvarez-Grande J: Pseudoaneurysm of the thyrocervical trunk complicating percutaneous internal jugular-vein catheterization for haemodialysis. Nephrol Dial Transplant 13: 1009-1011, 1998

17) Peynircioğlu B, Ergun O, Hazirolan T, Serter T, Uçar I, Cil B, Cekirge S: Stent-graft applications in peripheral nonatherosclerotic arterial lesions. Diagn Interv Radiol 14: 40-50, 2008

18) Srinivasan A, Lesiuk H, Goyal M: Spontaneous resolution of posttraumatic middle meningeal artery pseudoaneurysm. AJNR Am J Neuroradiol 27: 882-883, 2006

19) You CK, Whatley GS: Swan-Ganz catheter-induced pulmonary artery pseudoaneurysm: a case of complete resolution without intervention. Can J Surg 37: 420-424, 1994

Address reprint requests to: Tsuyoshi Ohta, M.D., Ph.D., Department of Neurosurgery, Kitano Hospital, 2-4-20 Ohgimachi, Kita-ku, Osaka 530-8480, Japan.

e-mail: t-oota@kitano-hp.or.jp 